\title{
Expression of ceramide synthase 3 in non-small cell lung carcinoma distinguishes
} squamous cell lung carcinoma from adenocarcinoma.

Shahan Mamoor, MS1

1'shahanmamoor@gmail.com

East Islip, NY USA

Non-small cell lung cancer, classified as adenocarcinomas or squamous cell lung carcinomas, is the major cause of cancer death in the United States and worldwide ${ }^{1}$. To understand the most significant transcriptional differences between adenocarcinomas and squamous cell lung carcinomas, we mined published microarray data from two separate studies ${ }^{2,3}$. We identified ceramide synthase 3 (CERS3) as a distinguishing transcriptional feature of squamous cell lung carcinomas, suggesting the biology of CERS3 may be relevant to the pathways critical for the development or maintenance of squamous cell lung carcinomas but not of adenocarcinomas. CERS3 expression was significantly correlated with prognosis of patients with NSCLC, as patients with low tumor expression of CERS3 possessed significantly longer median overall survival than those with high tumor expression of CERS3. These analyses will also provide novel tools for diagnostic approaches and for guidance of treatment regimens for a cancer with dismal outlook.

Keywords: ceramide synthase 3, CERS3, non-small cell lung cancer, adenocarcinoma, squamous cell lung carcinoma, systems biology of NSCLC, targeted therapeutics in NSCLC, targeted diagnostics in NSCLC. 
Non-small cell lung cancer is the single most common cancer in the United States and the most common cause of cancer death in the United States ${ }^{1}$. The two major types of nonsmall cell lung cancer are adenocarcinoma and squamous cell lung carcinoma4.

Treatment approaches and prognosis can differ based on whether a patient is diagnosed with adenocarcinoma or squamous cell lung carcinoma ${ }^{4}$, and current approaches to distinguishing adenocarcinoma from squamous cell lung carcinoma rely on time-consuming procedures involving immunohistochemical staining with multiple markers including TTF1, p40, p63, CK5 and/or CK7 and subsequent readout by pathologists who will proceed to label each section, in a binary fashion, as positive or negative for each marker ${ }^{5}$. To understand the molecular nature of adenocarcinomas and squamous cell lung carcinomas in an unbiased fashion and at the systems level, and to facilitate discovery of genes with utility in NSCLC diagnostics in a quantitative real-time workflow providing a quantitative and independent rather than qualitative measure based on pathologist interpretation, we performed comparative transcriptome analysis ${ }^{2,3}$ of the two major types of NSCLC tumors: adenocarcinomas and squamous cell lung carcinomas.

This blind, systems-level approach identified the gene encoding ceramide synthase 3 , CERS3, as among the genes most differentially expressed when comparing adenocarcinomas and squamous cell lung carcinoma tumors, with CERS3 expression distinguishing squamous cell lung carcinomas from adenocarcinomas, suggesting CERS3 could be a useful tool in NSCLC diagnostics.

\section{Methods}

We utilized datasets GSE $74706^{2}$ and GSE $33532^{3}$ for this comparative transcriptome analysis of adenocarcinomas and squamous cell lung carcinomas. GSE74706 was generated using Agilent-026652 Whole Human Genome Microarray 4x44K v2 technology; for this analysis, we used $n=10$ adenocarcinomas and $n=4$ squamous cell lung carcinomas, and the analysis was performed using platform GPL13497. GSE33532 was generated using Affymetrix Human Genome U133 Plus 2.0 Array technology; for this analysis, we used $n=10$ adenocarcinoma tumors and $n=8$ squamous cell lung carcinomas, and the analysis was performed using platform GPL570.

The Benjamini and Hochberg method of $p$-value adjustment was used for ranking of differential expression but raw $p$-values were used to assess statistical significance of global differential expression. Log-transformation of data was auto-detected, and the NCBI generated category of platform annotation was used. A statistical test was performed to evaluate whether CERS3 expression was significantly between adenocarcinoma and squamous cell carcinoma using a two-tailed, unpaired t-test with Welch's correction. We used PRISM for all statistical analyses of differential gene expression in NSCLC tumors (Version 8.4.0)(455). For Kaplan-Meier survival analysis, we used the Kaplan-Meier plotter online tool 7 for correlation of CERS3 mRNA expression levels with overall survival in $n=1925$ non-small cell lung cancer patients. 


\section{Results}

We mined published microarray data from two separate studies ${ }^{2,3}$ to describe in an unbiased fashion and at the systems-level genes whose expression was most specific to either one of the two major types of non-small cell lung cancer: adenocarcinomas and squamous cell lung carcinomas.

\section{CERS3 expression distinguishes squamous cell lung carcinomas from adenocarcinomas.}

By comparing the global gene expression profiles of squamous cell lung carcinomas to adenocarcinomas, we found that the ceramide synthase 3 was among the most differentially expressed genes between the two major sub-types of NSCLC 2 (Table 1). When sorting each of the genes expression by microarray based on significance of change in expression between adenocarcinomas and squamous cell lung carcinomas, CERS3 ranked 20 out of 34183 total transcripts (Table 1). CERS3 differential expression between adenocarcinomas and squamous cell lung carcinomas was statistically significant (Table $1 ; p=4.43 \mathrm{E}-09$ ).

In a separate dataset ${ }^{3}$, CERS3 was again amongst the most differentially expressed when comparing squamous cell lung carcinomas to adenocarcinomas in NSCLC (Table 2). When sorting each of the genes expression by microarray based on significance of change in expression between adenocarcinomas and squamous cell lung carcinomas, CERS3 ranked 18 out of 25906 total transcripts (Table 2). CERS3 differential expression in NSCLC when comparing adenocarcinomas and squamous cell lung carcinomas was again statistically significant (Table $2 ; p=4.34 \mathrm{E}-26$ ).

\section{Ceramide synthase 3 is expressed at significantly higher levels in squamous cell lung} carcinomas than in adenocarcinomas.

We obtained exact mRNA values for CERS3 to understand the magnitude and direction of difference in CERS3 mRNA expression between adenocarcinomas and squamous cell lung carcinomas in NSCLC. CERS3 was expressed at significantly higher levels in squamous cell lung carcinomas as compared to adenocarcinomas, and this difference was statistically significant (Figure 1 and Figure 2: $p<0.0001$ and $p=0.0047$, respectively). We calculated a mean fold change of $1.9592 \pm 0.2543$ in CERS3 expression when comparing squamous cell lung carcinomas to adenocarcinomas (Table 2).

\section{Expression of CERS3 is associated with patient survival in NSCLC.}

We performed Kaplan Meier survival analysis ${ }^{7}$ to determine if CERS3 expression was correlated with patient outcomes in NSCLC. We found significant correlation between expression of CERS3 and overall survival in patients with NSCLC (Figure 3 and Table 3); high CERS3 tumor expression was a negative prognostic indicator. While median overall survival was 112.67 months for NSCLC patients with low expression of CERS3, median overall survival was 54.57 months for NSCLC patients with high expression of CERS3. Correlation of CERS3 tumor expression with median OS with statistically significant (Figure 3; log rank $p$-value: 3.7e-08; hazard ratio: $1.6(1.35-1.89))$. 
Thus, by mining published microarray data from independent datasets, we found that CERS3 was among the genes whose expression most significantly distinguished squamous cell lung carcinomas from adenocarcinomas in NSCLC. Moreover, we found a statistically significant correlation between CERS3 tumor expression at the mRNA level and patient survival in NSCLC.

\section{Discussion}

To understand the most striking transcriptional features of each of the two major tumor types in NSCLC, we performed comparative transcriptome analysis of ACC and SCLC tumors using published microarray data, discovering ceramide synthase 3 as the among the most distinguishing transcriptional feature of squamous cell lung carcinomas.

Ceramide synthase 3, CERS3, was initially identified as the longevity assurance homologue (LASS) family member LASS3, encoding a protein of 384 amino acids in length as well as a longer variant 419 amino acids long termed LASS3-long, and initially reporter to possess expression mostly restricted to the testis but also at lower levels in the skin. Mass spectrometry of cells over-expressing LASS3/CERS3 revealed increases in ceramide species, particularly middle- to long-chain-fatty acyl-CoAs, suggesting CERS3 could function in biosynthesis of ceramides ${ }^{8}$. CERS3 is localized to the endoplasmic reticulum at the subcellular level and in the upper stratum spinous and stratum granulosum at the level of the tissue in the skin. The outer layer of the epidermis, the stratum corneum, is protected by the cornified envelope (CE); CE proteins are bound to ceramides with ultra-long-chain acyl groups (ULCCers) and ULC-Cers are also integral components of extracellular lipid lamellae (ELL). Human and mouse CERS3 uniquely functions in synthesis of ULC-Cers. Mice deficient in CERS3 manifest complete lack of ULC-Cers with carbon chain lengths greater than or equal to 26 , and both the ELL and cornified lipid envelope are non-existent or non-functional ${ }^{9}$. The phenotypic severity of CERS3 deficiency in mice is evidenced by death of mutant mice after birth due to water loss from the epidermis and susceptibility of mutant skin to Candida albicans yeast infections. The stratum corneum is nearly twice as thick (hyperkeratosis) ${ }^{9}$. Filipin and Nile red staining of cholesterol and lipids in the epidermis revealed an abnormal pearl-like distribution of lipids, supported disruption of the lamellar structure of the upper stratum corneum ${ }^{9}$. Moreover, while non-peripheral corneodesmosomes (CDs) are normally degraded in the outer layers of the skin, in mutant skin, CDs remained and were intact; desmoglein 1, a component of CDs, was maintained in mutant stratum corneum but undetectable in wild-type stratum corneum ${ }^{9}$. CERS3 deficiency caused by somatic mutation is associated with dysfunction and disease in humans as well. A microdeletion partially encompassing CERS3 was found in four patients with autosomal recessive congenital ichthyosis (ARCl); while the portion of the genome affected in these patients was not solely restricted to CERS3, further analysis revealed patients with nonsyndromic ARCI with splice site mutations in CERS3 ${ }^{10}$. In vitro evaluation of patient skin revealed that CERS3 mutations resulted in abnormal sphingolipid profiles with decreased levels of very long-chain ceramides in the epidermis ${ }^{10}$. 
We could not identify literature describing a role for ceramide synthase 3 in non-small cell lung cancer. However, it may be of relevance to note that daunorubicin, an anthracycline, induces cell death by apoptosis in P388 cells; this induction of cell death is accompanied by increased levels of intracellular ceramide. Fumonisin B1, a natural product with structural similarities to sphingosine, functions as an inhibitor of ceramide synthase and critically, fumonisin B1 could block both generation of ceramide and induction of apoptosis by daunorubicin ${ }^{11}$. Together these data suggested that the chemotherapeutic anthracycline daunorubicin could induce cell death through generation of ceramides ${ }^{11}$. Ceramides have been associated with induction of cell death in at least one human cancer. In mantle cell lymphoma (MCL) cells, cannabinoid treatment could induce increased levels of ceramides, and treatment of the Rec-1 MCL cell line with the endocannbinoid analogue $R(+)$-methanandamide (R-MA) resulted in increased ceramides $\left(\mathrm{C}_{16}, \mathrm{C}_{18}, \mathrm{C}_{24}\right.$ and $\mathrm{C}_{24: 1}$ species) and induction of CERS3 and CERS6 gene expression. Depletion of CERS3 and CERS6 blocked generation of $\mathrm{C}_{16}$ and $\mathrm{C}_{24}$ by R-MA, supporting the notion that cannabinoids could induce ceramide synthesis in MCL cells. Importantly, R-MA treatment resulted in induction of cell death, and inhibition of ceramide synthesis with fumonisin B1 or another ceramide pathway inhibitor myriocin resulted in significant abrogation of cell death induced by R-MA, together arguing that cannabinoids could induce cell death through ceramide synthesis in MCL cells ${ }^{12}$.

We found that the ceramide synthase 3 was the among the most differentially expressed gene when comparing the tumors of patients with the two most common sub-types of NSCLC: adenocarcinomas and squamous cell lung carcinomas. We also found significant correlation between overall survival of NSCLC patients and CERS3 expression. CERS3 has value as a diagnostic tool, as a prognostic indicator, and the biology of CERS3 may be of value in understanding fundamental differences between the two major types of the most common type of cancer, and the most common cause of cancer death in the United States and worldwide. 


\section{References}

1. Siegel, R.L., Miller, K.D. and Jemal, A., 2019. Cancer statistics, 2019. CA: a cancer journal for clinicians, 69(1), pp.7-34.

2. Marwitz, S., Depner, S., Dvornikov, D., Merkle, R., Szczygieł, M., Müller-Decker, K., Lucarelli, P., Wäsch, M., Mairbäurl, H., Rabe, K.F. and Kugler, C., 2016. Downregulation of the TGF $\beta$ pseudoreceptor BAMBI in non-small cell lung cancer enhances TGF $\beta$ signaling and invasion. Cancer research, 76(13), pp.3785-3801.

3. Kabbout, M., Garcia, M.M., Fujimoto, J., Liu, D.D., Woods, D., Chow, C.W., Mendoza, G., Momin, A.A., James, B.P., Solis, L. and Behrens, C., 2013. Ets2 mediated tumor suppressive function and met oncogene inhibition in human non-small cell lung cancer. Clinical cancer research, 19(13), pp.3383-3395.

4. Cetin, K., Ettinger, D.S., Hei, Y.J. and D O'Malley, C., 2011. Survival by histologic subtype in stage IV nonsmall cell lung cancer based on data from the Surveillance, Epidemiology and End Results Program. Clinical epidemiology, 3, p.139.

5. Yuan, M., Huang, L.L., Chen, J.H., Wu, J. and Xu, Q., 2019. The emerging treatment landscape of targeted therapy in non-small-cell lung cancer. Signal Transduction and Targeted Therapy, 4(1), pp.1-14.

6. Osmani, L., Askin, F., Gabrielson, E. and Li, Q.K., 2018, October. Current WHO guidelines and the critical role of immunohistochemical markers in the subclassification of non-small cell lung carcinoma (NSCLC): Moving from targeted therapy to immunotherapy. In Seminars in cancer biology (Vol. 52, pp. 103-109). Academic Press.

7. Gyorffy, B., Surowiak, P., Budczies, J. and Lanczky, A., 2013. Online survival analysis software to assess the prognostic value of biomarkers using transcriptomic data in non-smallcell lung cancer. PloS one, 8(12), pp.e82241-e82241.

8. Mizutani, Y., Kihara, A. and Igarashi, Y., 2006. LASS3 (longevity assurance homologue 3) is a mainly testis-specific (dihydro) ceramide synthase with relatively broad substrate specificity. Biochemical Journal, 398(3), pp.531-538.

9. Jennemann, R., Rabionet, M., Gorgas, K., Epstein, S., Dalpke, A., Rothermel, U., Bayerle, A., van der Hoeven, F., Imgrund, S., Kirsch, J. and Nickel, W., 2012. Loss of ceramide synthase 3 causes lethal skin barrier disruption. Human molecular genetics, 21(3), pp.586-608.

10.Radner, F.P., Marrakchi, S., Kirchmeier, P., Kim, G.J., Ribierre, F., Kamoun, B., Abid, L., Leipoldt, M., Turki, H., Schempp, W. and Heilig, R., 2013. Mutations in CERS3 cause autosomal recessive congenital ichthyosis in humans. PLoS Genet, 9(6), p.e1003536. 
11.Bose, R., Verheij, M., Haimovitz-Friedman, A., Scotto, K., Fuks, Z. and Kolesnick, R., 1995. Ceramide synthase mediates daunorubicin-induced apoptosis: an alternative mechanism for generating death signals. Cell, 82(3), pp.405-414.

12.Gustafsson, K., Sander, B., Bielawski, J., Hannun, Y.A. and Flygare, J., 2009. Potentiation of cannabinoid-induced cytotoxicity in mantle cell lymphoma through modulation of ceramide metabolism. Molecular cancer research, 7(7), pp.1086-1098. 


\section{CERS3}

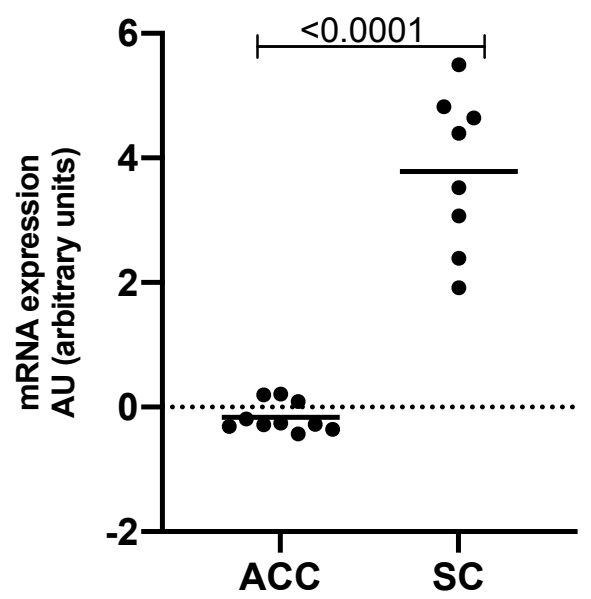

Figure 1: Expression of ceramide synthase 3 distinguishes squamous cell lung carcinoma from adenocarcinoma.

Messenger RNA (mRNA) levels of CERS3 in the tumors of patients with adenocarcinoma ("ACC"; left) and in the tumors of patients with squamous cell lung carcinoma ("SC"; right) are graphically represented here with mean mRNA levels marked and the result of a statistical test evaluating significance of difference in mRNA expression between the tumors of patients with adenocarcinomas and squamous cell lung carcinomas, a $p$-value, listed above. 


\section{CERS3}

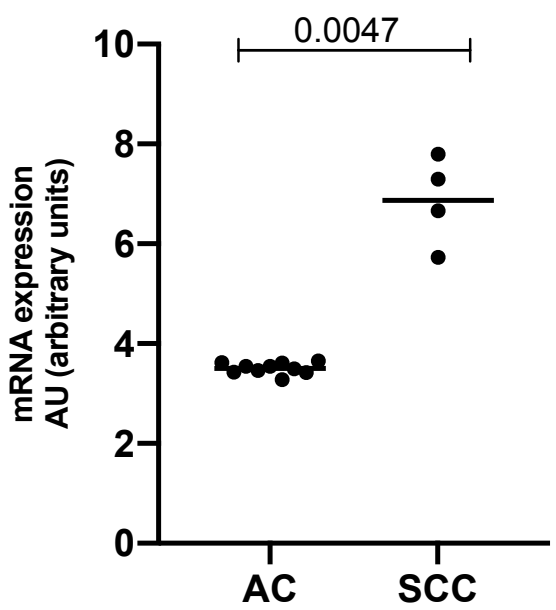

Figure 2: Expression of ceramide synthase 3 distinguishes squamous cell lung carcinoma from adenocarcinoma.

Messenger RNA (mRNA) levels of CERS3 the tumors of patients with adenocarcinoma ("ACC"; left) and in the tumors of patients with squamous cell lung carcinoma ("SC"; right) are graphically represented here with mean mRNA levels marked and the result of a statistical test evaluating significance of difference in mRNA expression between the tumors of patients with adenocarcinomas and squamous cell lung carcinomas, a $p$ value, listed above. 


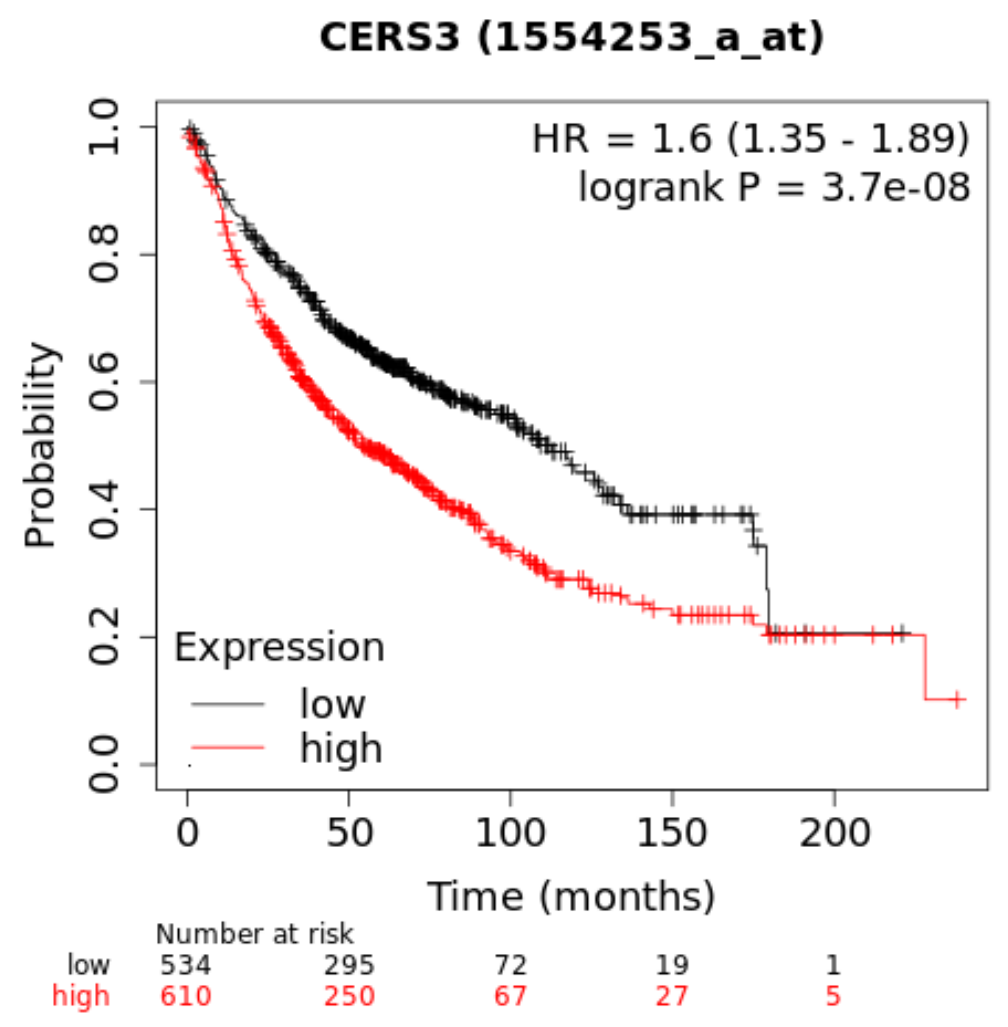

Figure 3: Ceramide synthase 3 expression in the tumors of patients with NSCLC correlates with overall survival.

Depicted in this Kaplan-Meier plot is the probability of overall survival for $n=1925$ total patients stratified into two groups, based on low or high expression of CERS3 in patient tumors. The log rank $p$-value denoting statistical significance of difference in overall survival when comparing the two groups, as well as hazard ratio for this comparison is listed above. Listed below is the number of patients at risk (number of patients alive) per interval, after stratification based on CERS3 expression; in the first interval, number at risk is number of patients alive; in each subsequent interval, number at risk is the number at risk less those who have expired or are censored. 
Table 3: Median overall survival of NSCLC patients with low tumor expression of CERS3 is significantly greater than in patients with high tumor expression of CERS3.

The median overall survival of $n=1925$ NSCLC patients based on stratification into low or high expression of CERS3 in tumors is listed in this chart. 